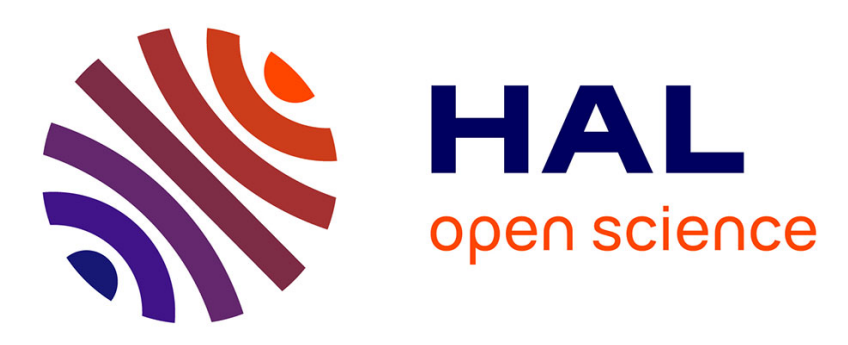

\title{
Des techniques pour analyser le patrimoine archéologique monumental : le cas du temple d'Opet à Karnak
}

Emmanuel Laroze

\section{- To cite this version:}

Emmanuel Laroze. Des techniques pour analyser le patrimoine archéologique monumental: le cas du temple d'Opet à Karnak. Bulletin - Société Française de Photogrammétrie et de Télédétection, 2011, 196, p.59-64. 10.52638/rfpt.2011.38 . hal-01061659

\section{HAL Id: hal-01061659 \\ https://hal.science/hal-01061659}

Submitted on 8 Sep 2014

HAL is a multi-disciplinary open access archive for the deposit and dissemination of scientific research documents, whether they are published or not. The documents may come from teaching and research institutions in France or abroad, or from public or private research centers.
L'archive ouverte pluridisciplinaire $\mathbf{H A L}$, est destinée au dépôt et à la diffusion de documents scientifiques de niveau recherche, publiés ou non, émanant des établissements d'enseignement et de recherche français ou étrangers, des laboratoires publics ou privés. 


\section{Revue}

\section{Française de}

Photogrammétrie et de Télédétection

$n^{\circ} 196$

Photogrammétrie au service des archéologues et des architectes

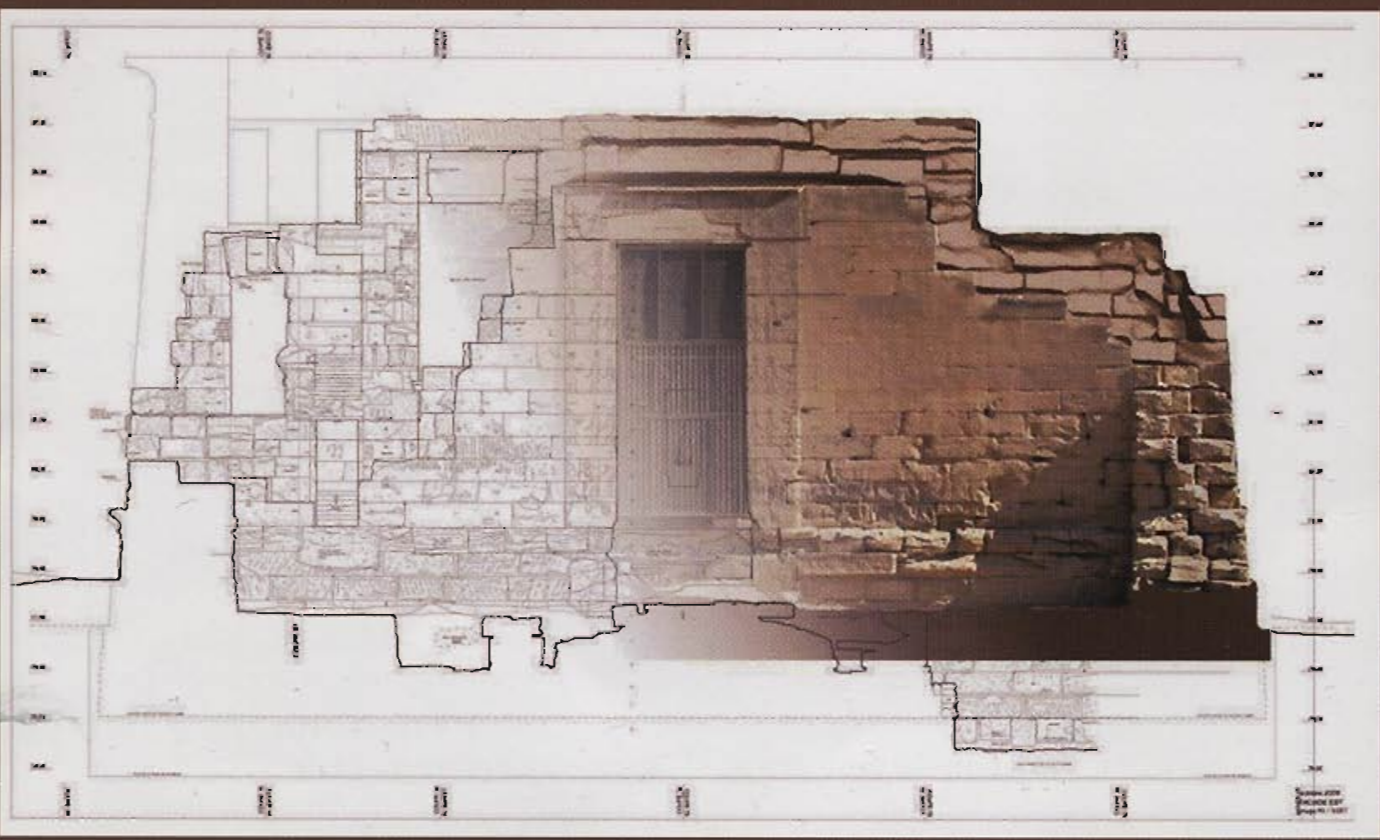

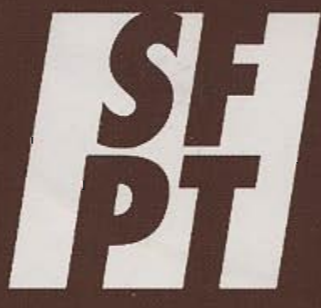

Société

Française

de Photogrammétri et de Télédétection

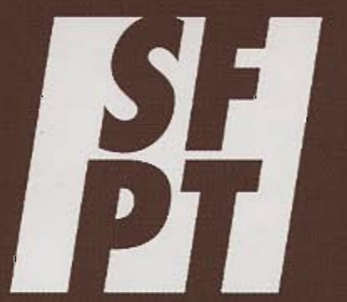

Société

Française

de Photogrammétrie et de Télédétection

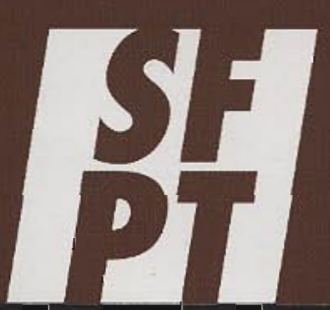




\section{Revue Française de Photogrammétrie et de Télédétection $n^{\circ} 196$}

\section{Sommaire}

Editorial

Ruphä̌le Héno

Valorisation numérique du théâtre Gallo-Romain de Mandeure

Emmannel Alby, Eddy Smigiel, Pierre Assali

Photogrammétrie pour la recherche architecturale : vingt ans d'expérience à l'Ecole d'Eudes Arabes

Antomio Almagro

La citerne El-Nabih à Alexandrie : un exemple d’utilisation de la photogrammétrie, de la lasergrammétrie et du modèle 3D sur un chantier de fouille archéologique

Lawrent Borel

Plorogrammétrie et photographies : des outils complémentaires pour la reconstitution numérique du patrimoine. L'exemple de la modélisation des toits de deux bâtiments de terre de la ville de Bam (Iran)

Olivier Bonet. Majid Beqqali, Sahar Ghaderi, Franck Chopin

Une interface ergononique de calcul de modèles 3D par photogrammétrie lsabelle Cléry. Marc Pierrot-Deseilligny.

De l'acquisition 3D à la téalité augmentée : le cas de l’église de la chartreuse pontificale de Villeneuve-lèsAvignon (Gard)

Aurélie Fave-Brun, Livio de Luca

Des techniques pour analyser le patrimoine archéologique monumental : le cas du temple d’Opet à Karnak Emmanuel Laroze 


\title{
DES TECHNIQUES POUR ANALYSER LE PATRIMOINE ARCHÉOLOGIQUE MONUMENTAL : LE CAS DU TEMPLE D’OPET À KARNAK
}

\author{
Emmanuel Laroze \\ Laboratoire Orient et Méditerranée - U.M.R. 8167 du CNRS Département Monde Pharaonique / Université Paris \\ Sorbonne 1, rue Victor Cousin 75230 Paris Cedex 05 \\ laroze.emmanuel@gmail.com
}

\begin{abstract}
Résumé
Les techniques appliquées au relevé dans le domaine des études architecturales, ne cessent de se perfectionner. L'archéologue du bấti dispose de nos jours des moyens variés que sont la phologrammétrie, la lasergrammétrie, et tout autant le dessin traditionnel. La capacité à produire plus rapidement une documentation extrêmement précise, implique néanmoins quelques changements en termes de méthodologie. Les nouvelles techniques de relevé tendent à produire des images plus fidèles au sujet -que l'on pourra penser plus "objectives"- mais pourraient s'éloigner de leur vocation analytique initiale : si le dessin manuel organisait les données tout en les enregistrant, l'automatisation partielle des technologies ne permet pas d'opérer cette sélection première de l'information. Il est par conséquent intéressant de se questionner sur leur pertinence, en fonction des contextes et des programmes. L'étude architecturale menée sur le temple ptolémaïque d'Opet à Karnak en Haute-Égypte a permis d'apporter quelques réflexions sur le sujet. Le relevé complet du monument a mis en œuvre différentes techniques telles que le scannage 3d ou le redressement de photographies. Leur utilisation sur ce chantier archéologique contribue à dresser une réflexion critique sur leur utilisation et leur efficacité.
\end{abstract}

Mots clés : Étude architectural, photogrammétrie, lasergrammétrie, redressement photographique.

\begin{abstract}
The techniques applied to the architectural studies are constantly developed and enhanced. According to the context, the archaeologist can choose from among technical tools based on photogrammetry, lasergrammetry or even technical drawing. Nowadays, the complete survey of a building is easier and faster than ever. Nevertheless, the possibility to capture reality "as-built" leads to the production of more "objective" documents than before. Indeed, architectural survey can be traditionally defined as a method to identify and gather information about historic architectural resources. Usually. the drawing consists to select information which must be recorded. How are applied the different techniques in the case of architectural studies? What are their interactions? Some answers can be finding in a recent work: the study of the antique Opet temple at Karnak. The analysis of the monument has requested a complete and accurate survey. Various techniques and tools have been employed: $3 d$ scanner, digital photographic rectification, drawings... Throw this experience it's possible to give a critical point of view of their application in the field of archaeology.
\end{abstract}

Keywords : Architectural study, photogrammetry, 3D laser scanning, rectified photography.

"Croyez-vous que la photographie peut dispenser de dessiner, non pour le plaisir d'amasser des images dans des portefeuilles, mais parce que la pratique du dessin habitue les yeux à voir plus vite, plus juste et mieux, en établissant entre l'organe de la vision et le cerveau une sorte de travail en commun qui tacilite les déductions ? Dix fois nous passons devant un objet et le regardons attentivement parce qu'il nous intéresse ; nous croyons le connaître parfaitement dans sa forme générale comme dans ses détails. Un jour, il nous prend l'idée de le dessiner, et nous lui découvrons des qualités que nous ne soupçonnions pas, malgré notre ferme volonté de bien observer". Eugène Viollet-le-Duc, 1879. Histoire d'un dessinateur, Comment on apprend à dessiner, Paris, France p.66.

\section{Introduction}

En matière d'élude architecturale, le vestige est la source d'information "première". II peut constituer à lui seul une somme documentaire "suffisante". Pourtant en traversant les siècles, le monument s'est érodé, transformé voire ruiné; selon son étal de conservation, il aura préservé tout ou partie de son histoire. On pout dire qu'il s'agit de "témoins" plus ou moins complets, que l'architecte tentera de faire parler. Dans le cas précis du patrimoine antique, on s'attardera d'autant plus sur l'étude de ces documents "en dur", qu'ils constituent fréquemment l'unique source d'information. En effet nous recensons peu de textes datés de ces époques et concernant le báti, qu'ils n'aient pas existé ou qu'ils ne nous soient pas parvenus. L'observation et 
les fouilles archéologiques sont alors les seuls moyens d'investigation laissés au chercheur. Sa táche consistera à interpréter le vestige "de visu", en s'appuyant sur quelques rélérentiels. Pour mener son analyse, il dispose d'une méthode fondée sur l'observation, la prise de note et le dessin, que l'on appelle le "relevé".

\section{Le relevé}

\subsection{Trler l'information}

Le relevé consiste toujours à opérer une série de choix dans la masse d'informations à archiver. Sur le lerrain, il reste une opération spécifique, qui ne se confond pas dans la seule acquisition de données. II s'agit d'une veritable "méthode d'analyse" graphique, que l'architecte ou l'archéologue mel en ceuvre pour trier puis organiser les données ; il écartera d'emblée celles qu'il jugera inutiles à l'étude. A travers ce processus de sélection, on pratique une sorte d'appauvrissement de l'information qui, loin de desservir le document, permet d'en dégager l'essentiel: comme l'écrit René Ginouvès, "une image est d'autant plus tacile à utiliser qu'elle a davantage perdu d'information" (Ginouvès, 1987).

A partir des "minutes de terrain"', sera réalisé un ensemble de documents synthétiques, qui lacilitera la confrontation des données diverses. En règle générale et quelle que soit la technique mise en ceuvre, le relevé aboutit in fine à une documentation relativement normalisée de plans et de coupes, qui rendra visible la globalité de l'édifice à une même échelle réduite, sous l'angle conventionnel de vues frontales. C'est celte harmonisation de l'information qui permettra d'établir des relations spatiales entre les différents éléments architecturaux. Le dessin en coupe par exemple, facilitera la comparaison des altitudes et pourra mettre au jour certaines relations spatiales ou constructives entre telle et telle piece. Un plan quant à lui, éclairera le chercheur sur l'organisation structurelle et la composition précise des espaces. Par ailleurs, c'est sur cette même documentation initiale, que se baseront les premières hypothèses de restitutions architecturales. En entreprenant le relevé d'un édifice, l'architecte réalise, au fond, une sorte anamnèse ou projet des constructeurs antiques: dans l'élaboration de la documentation graphique, réapparaissent progressivement le plan originel du monument, ainsi que les méthodes et les techniques qui ont prévalu tant à sa conception qu'à sa fabrication. Enfin, dans le cadre d'une étude scientifique, la publication des relevés est indispensable car elle permet de "vérifier les démonstrations de l'auteur de la documentation, mais aussi o'en extraire des données utilisables pour d'autres études" (Zignani, 2008). Ces "planches" dessinees attestent de la rigueur de la démarche, appuient la démonstration, et valident la recherche.

${ }^{1}$ Ce terme désigne les relevés manuels ou les notes pris devant l'objẹt étudié.
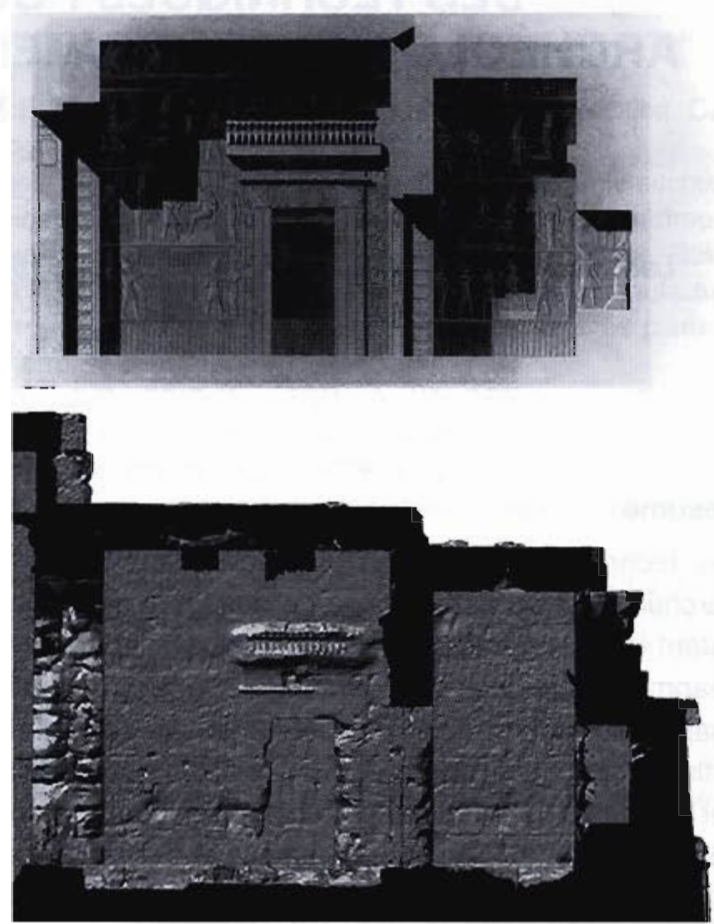

Figure 1 :La vue en géométral est un mode de représentation traditionnel en archilecture, utilisé aussı bien pour projeter un édifice que gour en faire l'èude. Deux sièdes séparent ces deux coupes longitudinales des salles centrales du temple d'Opet à Kamak. Malgré l'évolution des techniques de relevé, les codes de représentation demeurent les mêmes. En haut, lillustration liréx de la publication de la Description de l'Égyple Nol III, ol.59), montre un espace restltué , les ombres aident à la comprèhension du volume.

En bas, la projection frontale d'un modèle numérique, issu d'un scannage 3D, offre une vision plus objective du contexte, non séledive et sans interprètation (aAtm3d)

\subsection{Des codes de représentation}

Etonnamment, celte méthodologie propre à l'étude scientifique du báti n'a guère évolué depuis la naissance de la discipline archéologique. Les planches publiées dans la Description de l'Égypte (Jollois et Devilliers, 1821) par exemple, qu'il s'agisse des plans de masse. des coupes, ou des quelques détails rapportés, utilisaient des codes de représentations qui sont encore aujourd'hui les nôtres.

Le dessin a donc été d'emblée, un objet primordial pour l'archéologue du bâti, ef continue đ'avoir une place essentielle dans la construction de son analyse. Comme nous l'avons déjà signalé, les dispositils mis en ceuvre pour l'étude d'un patrimoine, auront tendance à "imiter" les méthodes de ses concepteurs. Ainsi, on peut supposer que la vue en géométral restera un outil d'analyse fondamental pour le chercheur, tant que les archilectes utiliseront ce même mode de représentation pour projeter leurs constructions. Si les procédés et les codifications du dessin n'ont guère évolué, les techniques directes ou indirectes appliquées à l'auscultation, à l'enregistrement de données ou à la production o'images ne cessent en revanche de se developper. Sans doute parce qu'elle est une discipline historique largement médiatisée el particulièrement propice à l'expérimentation, l'archéologie a toujours bénéficié assez directement de ces innovations technologiques. 


\subsection{Des outils pour reproduire le "tel quel"}

Là où jadis, l'architecte n'avait pour seuls outils que les traditionnels "papier, crayon, mètre, fil à plomb, niveau", il dispose désormais d'un large panel de technologies innovantes et complémentaires: la photogrammétrie, la photographie, la topographie, le redressement photographique, le scannage $3 d$, ou la photomodélisation, sont de plus en plus utilisés sur tes terrains archéologiques. L'application de ces dispositifs techniques, pour certains encore récents, aux contextes spécifiques du patrimoine, aura permis de développer de nouvelles procédures, en augmentant les capacités d'enregistrement: un maximum d'information peut être prélevé, avec une précision sans cesse accrue. L'utilisation d'un scanner $3 d$ par exemple, réduit le temps d'intervention sur le terrain à quelques jours, quand il fallait plusieurs semaines avec les outils traditionnels.

Ce même appareil permet d'acquérir des données autrefois difficilement accessibles, que l'on aurait alors renoncé à saisir; leur intérêt potentiel n'aurait pas suffi à justifier le temps consacré à leur acquisition. Ces différentes innovations ont également permis de dépasser un certain nombre de facteurs contraignants, comme les dimensions souvent monumentales des édifices. En effet, le relevé traditionnel se tait par "contaci": pour saisir une cole comme pour observer un détail, l'archéologue doit nécessairement se trouver à proximité de la paroi. Les équipements peuvent, du coup, être assez lourds; dans le cas des vestiges de grande hauteur, il faudra recourir à des échelles ou à de contraignants échafaudages. Avec l'essor des outils dérivẻs de la photogrammétrie ou de la lasergrammétrie, l'enregistrement volumique est devenu beaucoup plus pratique et rapide. La diffusion du numérique a d'ailleurs largement ouvert les champs d'application de la technique phologrammétrique, en démocratisant son utilisation. Avec la technique de corrélation d'image, 'appareil photographique numérique est aujourd'hui un outil performant, capable d'acquérir, en plus des couleurs, un ensemble d'informations volumiques. Les logiciels de photomodélisation s'étant développés parallèlement au matériel, l'exploitation des clichés photogrammétriques en est facilitée, tant du point de vue de l'auscultation (mesures de distances) que pour la fabrication de modèle numérique.

Ainsi, on observe une certaine évolution des pratiques, les principaux progrès concernant la rapidité et finalement la "commodité" d'acquisition. Des capteurs comme l'appareil photographique ou le scanner $3 d$ parviennent à prendre des mesures extrèmement précises, à distance. Cette sorte d'"éloignement" à l'objet d'étude est sans conteste un confort supplémentaire, qui augmente les possibilités d'investigation. En revanche, si la précision des instruments ne cesse de progresser, la qualité des données tend à s'uniformiser. Les informations ne sont plus hiérarchisées ; l'ensemble du monument est traité avec le même degré de définition: le global et le détail ne s'y différencient plus. La problématique en termes de méthode ne concerne donc pas tant la quantité ou la précision des éléments relevés, que leur "qualité" et leur possibilité d'exploitation. Brutes d'acquisition en quelque sorte, les données issues d'une majorité des "iechnologies nouvelles" produisent des représentations "objectives" d'un état du bâtiment. Celles-ci s'apparentent à des images photographiques et ont valeur de véritables "restitutions graphiques des formes architecturales" (De Luca, 2009) Cette capacité étonnante à reproduire le "tel quel", sera immédiatement exploitable dans les démarches d'archivage; elle permettra de produire des documents exacts, pouvant aussi servir de base graphique au dessinateur. Pourtant, cela ne suffit pas à faire de ces images, des relevés en tant que tels.

Certaines tâches sont accélérées et les nouvelles capacités d'auscultation ouvrent des possibilités de prospection inédites. Indéniablement, le foisonnement d'instruments nouveaux a un impact très fort sur les travaux de recherche. Alin d'en proposer une pratique maîtrisée, il est aujourd'hui indispensable d'interroger leurs performances, au sein des diftérents domaines d'étude. De quelles manières ces procédés lechniques seront-ils mis en œuvre dans le cas spécifique d'une analyse architecturałe? Quelles seront leurs interactions? Le programme mené depuis près de 5 ans au temple d'Opet à Karnak, apporte quelques éléments de réponses. L'éventail large des techniques appliquées au relevé du temple, parfois de façon expérimentale, permet en effet de proposer un regard critique sur leur emploi.

\section{3. ÉTUDE ET TECHNIQUES DE RELEVE : LE CAS DU TEMPEE D'OPET}

\subsection{Le contexte}

L'étude architecturale dédiée au temple d'Opet, sur le site de Karnak en Haute-Egyple, a pour ambition de retracer l'histoire de cet édifice "tardif" daté de l'époque ptolémaĩque (vers 150 av. J.C.), et d'en comprendre les méthodes de construction et de conception. Dans ce cadre scientifique là, un relevé complet et détaillé du monument a été entrepris. La totalité des parois, les hauteurs d'assises, les éléments constructifs, les cassures spécifiques, les contours des décors ont été dessinés et le cas échéant, complétés par des annotations. Dès le début de l'étude et compte tenu des dimensions relativement modestes du monument ( $23 \times 46 \mathrm{~m})$, il a été décidé de travailler au 1/20ème. Cette échelle de relevé suffisamment grande, aura permis de représenter les innombrables détails d'une architecture sophistiquée. Une fois mis au net, les plans pourront ainsi être édités au 1/50ẻme. Le choix initial de l'échelle est déterminant : il fixe approximativement les dimensions auxqueiles les dessins seront publiés, et surtout, définit un niveau de détail à partir duquel le chercheur devra raisonner. 


\subsection{Le scannage $3 D$}

Un prix décerné par l'Institut de France a permis au projet de bénéficier dès son démarrage en 2004, des services d'une équipe spécialisée en lasergrammétrie ${ }^{2}$. Après deux campagnes de scannage 3D sur le terrain, celle-ci a réalisé un modèle numérique complet du monument (Chazaly et Laroze, 2009). Dans notre cas, c'est l'exploitation de ce type de document, en trois dimensions et difficilement manipulable, qui aurait pu poser problème. L'utilisation d'un petit logiciel ${ }^{3}$ permettant de visualiser le modèle et o'en extraire différentes informations, aura réglé les difficultés pratiques: notamment, l'interface du programme alfiche les coordonnées des points visés, permet de sortir différents profils au format dxf, ou d'exporter des vues en géométral.

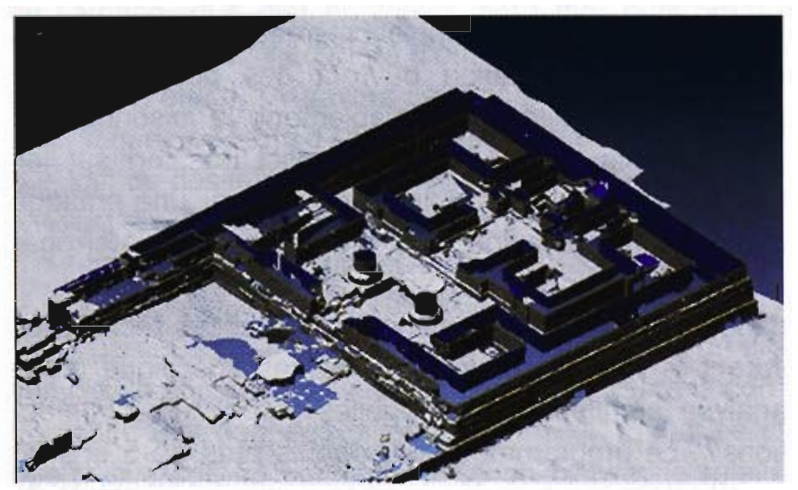

Figure 2 : Vue axonométrique coupée du temple d'Opet à Karnak. La manipulation d'un modèle numérique $3 \mathrm{~d}$ permet d'obtenir un nombre infini de vues sur l'objet: difiérents parametres sont combinables comme le point de vue, le plan ou le mode de projection (frontal ou en perspective). On peut ainsi éviter un certain nombre de déplacements sur le terrain. (C) Atm3d).

Il s'agit là d'une formidable base topographique, qui ne permet pourtant pas de représenter la totalité des détails -les joints des blocs par exemple-. Les images pholographiques associées systématiquement au modèle $30^{\circ}$ comblent ces quelques lacunes, et fournissent une information plus complète. Malheureusement, l'opération est complexe et les coùts de production des images restent élevés: quatre orthophotographies des seules parois extérieures ont été produites.

II s'agit là d'un type de représentation extrêmement précis et facile d'exploitation, qui s'apparente à un cliché photographique sans point de luite. Cette documentation partielle a complété le relevé détaillé de la totalité du temple, réalisé, lui, au moyen des techniques traditionnelles de dessin, avec le recours ponctuel à un théodolite. Une fois les "minutes" scannées et les déformations corrigées, les dessins ont été assemblés puis vectorisés à l'aide du logiciel Autocad.

\footnotetext{
2 www.atm3d.corn

${ }^{3}$ PolyWorks/Mview, www.innovmetric.com
}

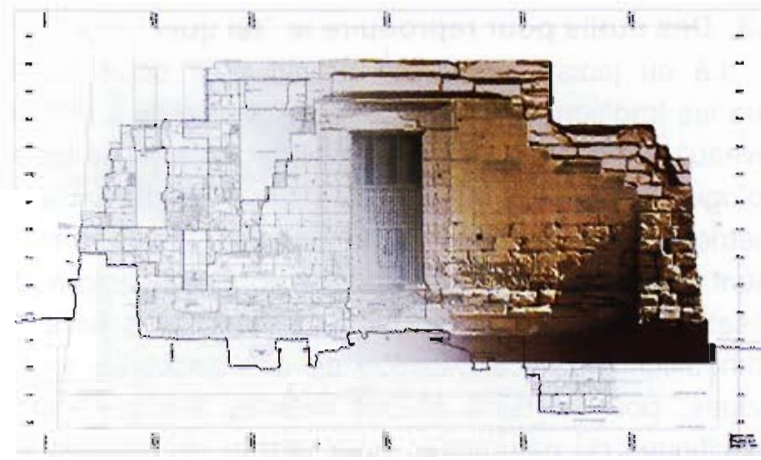

Figure 3 : Vue géométrale composite de la façade ouest du temple d'Opet à Karnak. Cette vue frontale confronte des informations variées, issues de trois techniques différentes. Le dessin au trait sur la partie de gauche de l'image, donne une vision plutót synthétique et analytique de l'édifice; elle est le résultat d'une série de choix opérés par le dessinateur. Lorthophotographie sur la droite, fournit une représentation plus "inmédiate" d'un état du bâtiment. Les données y ont été traitées techniquement, mais pas "éludiées". L'utilisation du modèle numérique brut en haut à droite, assure une bonne lisibilité des volumes. (C)E. Laroze /Atm3d)

\subsection{La photogrammétrie}

La phologrammétrie a permis de relever très efficacement les parois intérieures et d'en réaliser rapidement les élévations: le logiciel Redresseur développé par Yves Egels (IGN/ENSG) est en effet capable de produire l'image redressée d'une paroi plane, à partir de seulement quatre repères topographiques el au moyen d'un appareil photographique calibré.

Cette technique de redressement est particulièrement bien adaptée au contexte des temples égyptiens dont les murs, bien conservés et richement décorés, sont majoritairement plans. À Opet, l'outil photogrammétrique a permis de relever avec précision des informalions d'ordre tant constructif que décoratif, mettant ainsi en relation deux dimensions du temple trop souvent envisagées de laçon distincte. Par exemple, les profils des personnages ont pu étre positionnés sur les planches consacrées au relevé d'architecture, une présentation généralement absente des publications égyptologiques. L'objectif était double : proposer une description exhaustive du temple, et tenter de formuler des hypothèses inédites. Ainsi, l'acquisition d'informations précises liées aux décors a permis de comparer les différentes silhouettes des personnages aux contours de figures similaires. Ces confrontations "formelles" ont en quelque sorte stimulé la recherche, en remettant en chantier quelques "acquis" scientifiques: en effet, la superposition sur ordinateur des ligures, nous a permis de constaler que certaines parties - les contours des corps par exemple- se confondaient très exactement. Nous nous sommes donc dernandés quelles techniques ont été mises en cauvre, pour parvenir à un tel degré de précison. A partir de ces observations el quelques 


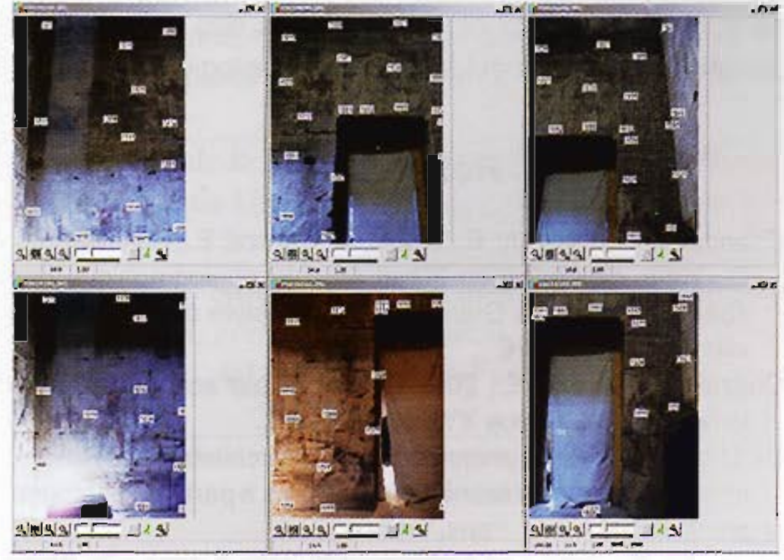

Figure 4 : Vue de l'interlace du logiciel Redresseur développé par Yves Egels. Une fois la couverture photographique réalisée, l'opération consiste a pointer des cibles dont les coordonnées sont connues, puis à faire correspondre divers points communs d'un cliché à l'autre (points de liaison). Après calcul, les images sont redressées indépendamment en fonction des paramètres de distorsion de l'objeclif.

autres, nous avons ainsi formulé l'hypothèse que certains éléments auraient pu être appliqués sur les parois à l'aide de poncifs, et non pas, comme cela est générale. ment admis, au moyen d'un carroyage. L'utilisation de gabarits par les anciens bàtisseurs, aurait permis en outre d'accélérer considérablement le Iravail de déco. ration el expliquerait l'aspect très normalisé des décors ptolémaïques. Cette application des outils de redressement photographique, montre de quelle façon l'utilisation de techniques émergentes peut renouveler les regards sur l'objet d'étude. En ce sens, l'expérimentation technologique fait partie intègrante de la recherche.

Lapplication complémentaire des différents procédés précédemment évoqués, produiront 25 coupes actuellement en cours d'élaboration, qui seront éditées à l'échelle du 1/50ème. L'ensemble des représentations assurera une couverture circonstanciée de toutes les parois ou temple avec, le cas échéant, le positionnement des principales figures du décor et des annolations.

\section{LA SPECIALISATION: UNE SCISSION DANS LE PROCESSUS}

L'arrivée de nouveaux outils d'auscultation, toujours plus performants mais aussi plus complexes ou "opaques", entraine inévitablement une spécialisation des pratiques. Des disciplines parfois très éloignées de la culture archéologique, font aujourơ'hui pleinement partie des processus de recherche: ingénieurs, topographes et pholographes sont de plus en plus souvent intégrés aux équipes, en tant que "professionnels" du relevé. Or, les formations et les prérogatives des uns et des autres sont parfois très éloignées. Loin de favoriser une pratique transversale de la recherche, cette pluridisciplinarité nouvelle aurait tendance à dissocier les compétences

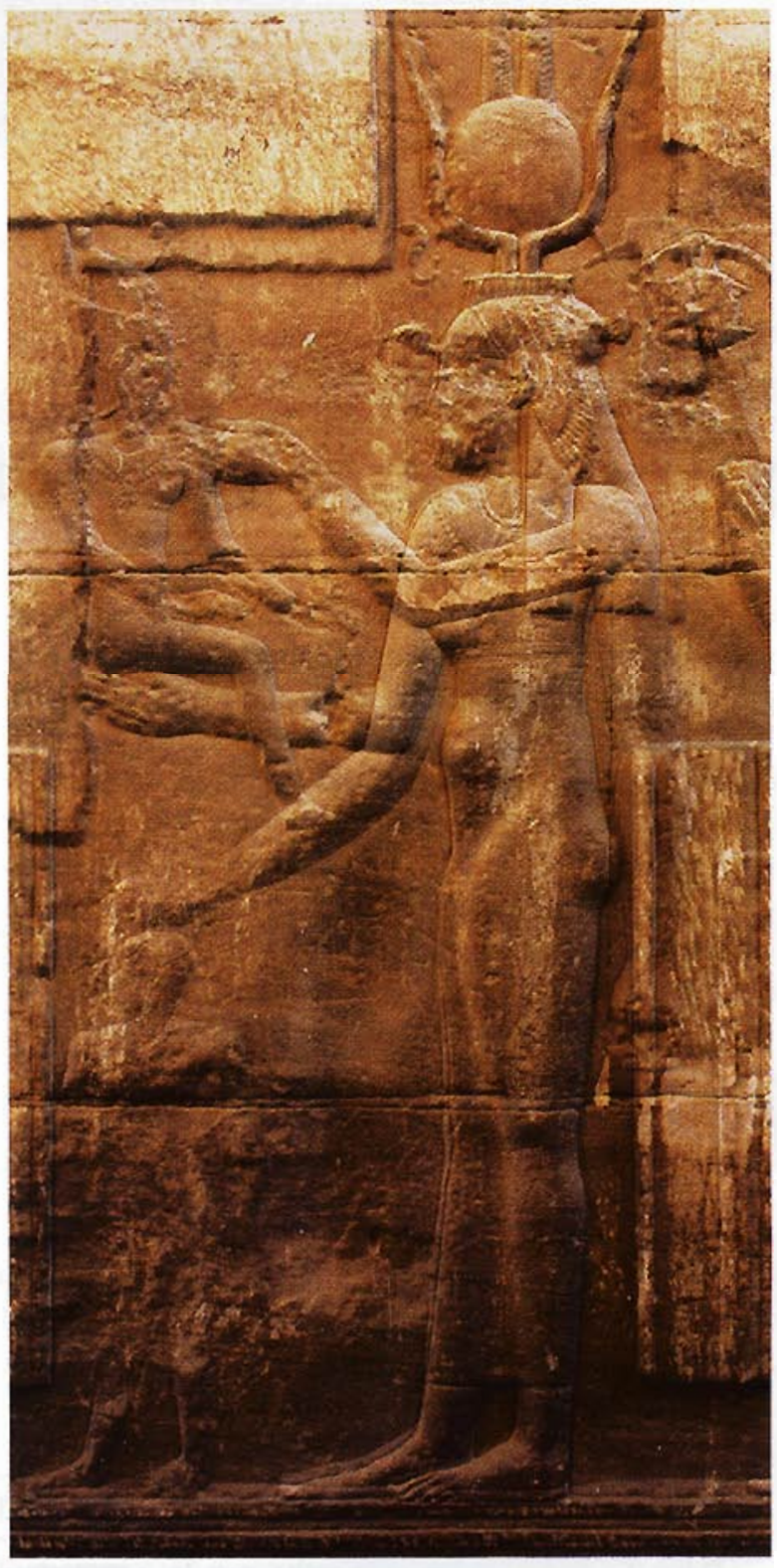

Flgure 5 : Superposition de deux figures. La technique du redressement photographique permet ici de confronter sur une méme image, deux représentations comparables d'une déesse du mammisi romain de Dendara. Les deux silhouetles sont ainsi "calées" très précisément l'une sur l'autre. On constate que la limite des corps et le visage des personnages correspondent parfaitement; seules les positions des bras difèrent. A partir de celte observation précise, on peut se demander si certains contours des figures n'étaient pas appliqués sur les parois avec l'aide de gabarits. (C)E. Laroze) 


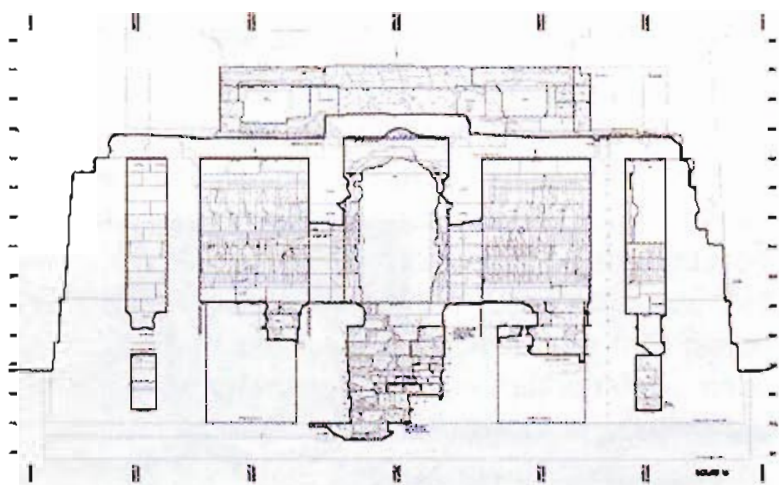

Figure 6 : Coupe transversale sur les salles centrales du temple d'Opet. Malgré l'homogénéité apparente du dessin au trait, la réalisation de cette image a demandé la mise en œuvre de plusieurs techniques de relevés : dessin tradítionnel, scannage 3d et photogrammétrie.(C)Alm3d).

de celui qui relève, "lingénieur", et de celui qui analyse, "l'archéologue". Le déroulement du récent programme d'étude des colonnes de la salle hypostyle de Karnak, illustre assez bien cette situation. La première étape, engagée depuis quelques années, a consisté à produire les déroulés des décors des 134 colonnes (Chandelier et al., 2009). Les ingénieurs topographes et les architectes ont été impliqués dans cette phase initiale, tandis que l'analyse sera réalisée dans un second temps par les égyptologues, à partir des résultats du relevé. Cette scission entre le fournisseur d'images et l'analyste, pourrait rapidement être contre-productive. $\mathrm{Si}$ "le mélange des genres" augure de formidables perspectives de recherche, il est aussi une difficulté à surmonter. II paraît indispensable que chacun des "experts" ne s'arrête pas à son domaine de spécialité, mais ouvre son champ de compétences. Cela pourra consister à expérimenter puis appliquer des techniques alternatives, ou simplement garder un esprit critique sur les objectifs communs. En effet, dans ce contexte inédit, l'échange et l'interaction entre les différents "producteurs" sont devenus nécessaires à la cohérence de la recherche et à la performance des résultats.

Dans le cadre d'une recherche sur le patrimoine archéologique monumental, "faire un relevé" consiste avant tout à analyser un objet, au moyen de techniques d'enregistrement. Celles-ci permettront de produire des représentations synthétiques du réel, traditionnellement en géométral. Le relevé est donc avant tout une méthode, permettant de questionner un vestige, de formuler et de vérifier des hypothèses. Les technologies, anciennes ou actuelles, restent des outils pour l'analyste, qui les emploiera variabiement, selon le contexte. Les programmes d'étude du bâti sont toujours contraints de diverses manières, et c'est bien ce contexte de recherche qui conduira à privilégier telle technique. II est ici important de souligner que les aspects pratiques et financiers, c'est-à-dire l'éconornie de moyen, reste un facteur déterminant dans le choix des outils de relevé. De ce point de vue la photogrammétrie a des perspectives de développements évidents en archéologie.

\section{Références}

Chandelier, L., Chazaly, B., Egels, Y., Laroze, E., Schelstraete, D. 2009. Numérisation 3D et déroulé photographique des 134 colonnes de la Grande Salle Hypostyle de Karnak. Revue XYZ 102, 21-26.

Chazaly, B., Laroze, E., 2009. Le relevé par scannage 3D du temple d'Opet. Revue XYZ 102, 21-26.

De Luca, L., 2009. La photomodélisation archilecturale. Relevé, modélisation et représentation o'édifices à partir de photographies. Ed. Eyrolles, Paris, France.

Ginouvès, R., 1987. Les techniques du relevé dans: 'le décor romain, les méthodes de releve et de restitution'. Dossiers Histoire et Archéologie 119 (7), 36-41.

Jallois, J., Devilliers, E., 1821. Description de l'Égypte. Ed. Panckoucke, Paris, France.

Zignani, P., 2008. Enseignement d'un temple egyptien, Conception architectonique du temple d'Hathor à Dendara. Presses polytechniques et universitaires romandes, Lausanne, Suisse. 